\title{
SIREN: Schema In-Fill Rendered from Entry Notifications
}

\author{
Elizabeth DeTienne, EMT; Milka Piszczek
}

POSTER PRESENTATION ABSTRACT | PROGRAM DEVELOPMENT \& EVALUATION CATEGORY

Introduction: Entry notes are radio communications between en-route first responders and a destination hospital to prepare hospital personnel for ambulance arrival. Ambulance services currently have no way to efficiently search entry note data or use it for training. The destination hospital may quickly lose the transmitted information, as it is reliant on a single listener. SIREN is an end-to-end tool that takes voice files of hospital entry notes as input and converts them into informative database tuples.

Program Development \& Implementation: SIREN takes in audio recordings of entry notes. The audio files are filtered using PySoX, by adding a low-pass filter, normalization, and a gain, which help remove background noise. The filtered audio is then transcribed using Google Cloud. From there, we extract the mentioned drugs using a dictionary of drug names, the chief complaints using a dictionary with associated symptoms and terminology, and the sex directly from the transcript. Parsing age is a non-trivial task since there's a large variance in the input (i.e. two-one vs. twenty one) and sometimes even repetition with the intent to clarify. We use Stanford's Natural Language Processing suite to extract cardinal numbers that are close to age-related words, and then we use a series of rules to extract the correct spoken age. We then put these into a database, which is visualized in a user-friendly table for skimming or searching.

Program Evaluation: We found that the audio transcript was sometimes cut short when a siren began in the background. Our filtering produced an incredible $50 \%$ increase in the median number of words transcribed. We manually verified the accuracy of a series of SIREN transcripts. Transcription accuracy was generally excellent, and we found Google's transcription errors to be our biggest bottleneck. A typical transcription has only minor mistakes that do not affect meaning. Leadership in MIT EMS noted that this would help elevate entry note skill, by giving EMTs a database they could easily and enjoyable peruse, or use to look back at their own performance.

Discussion/Conclusions: We see two potential applications for SIREN: (1) SIREN can train current EMS providers to give professional and useful entry notes, either on their peers' or their own entry notes; and (2) SIREN can be adapted to process data in real-time, creating a reference that hospital staff can use to improve continuity of care. We hope that this can be a useful tool for collegiate EMS in the future. Plans for future improvement include making SIREN real-time or using machine learning to train the audio-to-text with EMS-specific language.

Author Affiliations: Massachusetts Institute of Technology (MIT) Emergency Medical Services, MIT, Cambridge, MA, USA

Address for Correspondence: Elizabeth DeTienne, EMT.

E-mail: elizdet@mit.edu

Conflicts of Interest/Funding Sources: By the JCEMS Submission Declaration Form, all authors are required to disclose all potential conflicts of interest and funding sources. The authors declared that they have no conflicts of interest. The authors declared that they did not receive funding to conduct the program or research associated with this work.

Ethical Compliance: The authors attest that the research associated with this abstract was conducted in accordance with the JCEMS Ethics Guidelines.

Submission History: Received December 12, 2018; accepted for presentation and publication February 17, 2019.

Poster Presentation: This abstract was presented as a poster at the Academic Poster Session of the $26^{\text {th }}$ Annual Conference of the National Collegiate Emergency Medical Services Foundation; February 23, 2019; Pittsburgh, PA, USA. The authors received the Third Place Award for the Best Poster Presentation Competition.

Published Online: December 31, 2019

Published in Print: December 31, 2019 (Volume 2: Supplemental 1)

Reviewer Information: In accordance with JCEMS editorial policy, poster presen- tation abstracts undergo double-blind peer-review by at least two reviewers (JCEMS Editorial Board members and/or independent reviewers) prior to acceptance for presentation and publication. JCEMS thanks the anonymous reviewers who contributed to the review of this work.

Copyright: $\odot 2019$ DeTienne \& Piszczek. This is an open access abstract distributed under the terms of the Creative Commons Attribution 4.0 International (CC BY 4.0) License, which permits unrestricted use, distribution, and reproduction in any medium, provided the original author and source are credited. The full license is available at: https://creativecommons.org/licenses/by/4.0/

Electronic Link: https://doi.org/10.30542/JCEMS.2019.02.S1.03 